Simeonov, Plamen L.; Reschke, Dietrich :

Supporting adaptive multimedia with the wandering network model

Zuerst erschienen in:

Multimedia, image processing and soft computing : trends, principles and applications : proceedings of the Fifth Biannual World Automation Congress (WAC 2002), ISSCI 2002 and IFMIP 2002, Orlando, Florida, USA, Juni 09-13, 2002, (TSI Press Series Vol.13). Albuquerque, NM : TSI Press, 2002. - ISBN 1-88933-518-5, S. 319324

DOI: $\underline{10.1109 / W A C .2002 .1049563}$ 


\title{
SUPPORTING ADAPTIVE MULTIMEDIA WITH THE WANDERING NETWORK MODEL
}

\author{
Plamen L. Simeonov, Dietrich Reschke \\ Technische Universität Ilmenau \\ Fakultät füt Informatik und Automatisierung \\ Institut für Praktische Informatik und Medieninformatik \\ Email: \{plamen.simeonov |reschke\}@prakinf.tu-ilmenau.de
}

\section{ABSTRACT}

The Wandering Network [1] is a new type of communications architecture defined by:

1. flexible, multi-modal specialization of network nodes as virtual subnetworks;

2. mobility and virtualization of the net functions in hardware und software;

3. self-organization as multi-feedback-based topology-on-demand.

Network elemens can contain several exchangeable modules capable of executing diverse network functions in parallel. They can be invoked, transported to or generated in the nodes upon delivery of mobile code containing programs about the node's behaviour.

KEYWORDS: Adaptive Systems, Configurable Computing, Active Networks, Multimedia Architectures, Ad-hoc Mobility, Autopoiesis.

\section{INTRODUCTION}

Active networks (AN) has been a subject of intensive empirical investigation for the last decade. A number of different models have been proposed to implement active network architectures. Three major schools have been established until now:

1. The "programmable switch" model: User code is downloaded out-of band into the network nodes and executed on the normal flow packets treated as the code's data.

2. The "capsule" model: Each packet is treated as a complete program to be executed at each node of the network being traversed by the packet.

3. The "option" model: Some standard services or modules are residing in the network nodes. These are selected and invoked through options carried in the user's packet. The rest of the user's packet will be treated as data to be processed by the invoked routine.

Most AN approaches investigate and implement to some detail the above technical issues within a specific solution. Only a few survey papers were published trying to provide directions and goals for engineering within the field ([2], [3], [4]). These efforts were mainly focused on defining a common programming model of active networks. 
Recently, an integration and consolidation of the several different AN engirts: approaches can be observed. This trend is particularly evident at technology frontiers such as deeply embedded networked systems, autonomous software, configurable computing, adaptive systems, etc. However, implementations have shown that every single network issue such as caching, routing, management, etc. can have a specific active network solution. A number of requirements have been collected to activate the network. Yet, there is still no general recipe to address all the problems with only one end-to-end active network. The "killer" network of the future has not been found yet.

Along with the growing scope and number of ad-hoc solutions to active networking, the demand for their systematic categorization, evaluation and integration within a common research framework becomes increasingly evident. In particular, an evolutionary approach to active networking requires the development of common models for: a) the encoding of network programs in terms of mobility, safety and efficiency; b) the description and allocation of node resources; c) the built-in primitives and behavioral patterns available at each node.

We regard networking as a synthetic science. Therefore, the goal of this work is to provide a model-based theory, the Wandering Logic Intelligence (WLI), for the design and verification of evolutionary, autopoietic (self-creating) architectures, [5], based on active networks, reconfigurable computing [6], and adaptive systems, [7]. These three research fields were brought together for the following reasons. Firstly, active networking defines the principle and the goal of our research. Secondly, reconfigurable computing brings up the required detail and understanding within a context. Thirdly, adaptive systems encompasses the large field of heuristic techniques in AI for the purpose of organizing and optimizing wandering media communications. Finally, the application field of multimedia communications provide a challenging perspective on applying the WLI approach to the design and verification of autonomous adaptive architectures, [8].

\section{THE WLI MODEL}

Table 1 summarizes the basic characteristics of the ANTS active network architecture [9] along with the available options for extension (underlined) within WLI framework..

\begin{tabular}{|l|l|}
\hline Active Nodes & Active Packets \\
\hline - $\begin{array}{l}\text { Have structure that could be re-configured } \\
\text { with time. }\end{array}$ & $\begin{array}{l}\text { Have structure that could be re-configured } \\
\text { with time. }\end{array}$ \\
- $\begin{array}{l}\text { May accommodate some residential program } \\
\text { code for processing packets. Could support } \\
\text { multiple code schemes to define classes of }\end{array}$ & $\begin{array}{l}\text { May carry program code, but do not } \\
\text { execute it. Could support multiple code } \\
\text { schernes. Could carry some code for AN }\end{array}$ \\
$\begin{array}{l}\text { services. } \\
\text { Do processing on packets. Could be } \\
\text { processed by packets. Could do some }\end{array}$ & $\begin{array}{l}\text { configuration. } \\
\text { processing on themselves. }\end{array}$ \\
\hline $\begin{array}{l}\text { Could be mobile. } \\
\text { processing on nodes. Could do some }\end{array}$ \\
\hline
\end{tabular}

Table 1: Possible enhancements to the concept of active networks. 
In [1] we defined a new network generalization for programmable active networks which we call the Wandering Network (WN). The new concept is based on previous research in intelligent and smart networking ([10], [11]) and a fomalism called $W L I$ (the Wandering Logic Intelligence) [12] which extends the Columbia Model of a programmable network, [4], by three essential characteristics:

1. it is a real active network which means that it is truly programmable and reconfigurable, including the network hardware up to the gate level;

2. it is a runtime extensible and exchangeable network in terms of both software and hardware components, i.e. a wandering network;

3. it is an evolutionary network which realizes adaptive self-distribution and replication of sub-networks:

a. by guided or autonomous node and component mobility in terms of hardware;

b. by including essential engineering information in the mobile code of the active packets and applying genetic transcoding mechanisms in the active nodes (netbots).

The elements of a WLI architecture have a temporal character; they can be created, configured and removed (on demand). Thus, in a Wandering Network, functions can change their hosts, wander and settle down in other hosts, thus creating a valuable statistics about the frequency of usage of wandering functions in the network.

The WLI model comprises a self-organizing mobile active network with both nodes (netbots) and packets (shuttles) being active (i.e. executable), exchangeable, reconfigurable and programmable down to the level of gate components. A netbot processing the shuttle contents can change its state and re-configure its resources and links to perform accordingly. It can also change the state of the shuttle. On the other hand, a shuttle approaching a netbot can reconfigure itself becoming a morphing packet to provide the desired interface and match a netbot's requirements.

The Wandering Logic Intelligence (WLI) represents a hybrid approach to active networking. It is an open, hierarchical and dynamically structured model which allows to address specific problems in communication architectures and multimedia applications with a great degree of differentiation and flexibility in terms of software and hardware.

WLI generalizes AN capsules in shuttles as relatively autonomous mobile components including both programs and data possibly encoded in a language with corresponding references to nodes and other shuttles within the same or a different flow (protocol). This language is a generalization allowing us to address in a uniform way such issues as hypermedia content (e.g. MPEG-4) and knowledge management information systems as the one in [13] along with the corresponding encoding/decoding routines or references to them in some active network nodes or protocols. 
The following characteristics differentiate a Wandering Network from the traditional active and programmable network approaches:

- Active nodes may be mobile, and re-configurable (in terms of software and hardware). In addition to traditional active nodes, netbots can be also modified by shuttles. For this reason, the capsule APIs and the execution environments can be extended by special routines allowing the accommodation and execution of code that changes a netbot's configuration and resources. In this way, new functionality can not only be delivered to and injected into the node, but also distributed and optimized throughout the node itself. The runtime reconfiguration of a netbot can be invoket by internal procedures or upon execution of newly arrived shuttles.

- Active packets are called shuttles and carry code and data for the upgrade/degrade and re-configuration of netbots. Furthermore, the network protocol itself can be particularly embedded within the shuttle. Thus shuttles can carry genetic information about the nodes.

- A code distribution mechanism ensures that shuttle processing routines are automatically and dynamically transferred to the active nodes/netbots where they are required. In WLI, code distribution throughout the network and inside the nodes/netbots can be maintained by the shuttles themselves.

- The WLI formalism allows the creation of new shuttles (or the replication of "old" ones) in the intermediate active nodes under the supervision of the NodeOS. Furthermore, special classes of shuttles are allowed to replicate themselves and to create/remove/modify other shuttles and network resources.

\section{DISCUSSION}

The Wandering Logic of Intelligence (WLI), is a theory for modelling active and configurable mobile networks. Our preliminary goals are summarized as follows:

- to provide a formal means for the specification and verification of the generic temporal properties of active mobile nodes and packets;

- to support the reflexive dynamic adaptation of both mobile code (software) and node architecture (software and hardware);

- to provide the formal means for specification and verification of dynamic QoS and routing properties in ad-hoc mobile networks at both application (service) and packet level;

- to assist the formal transformation of the systems properties into mobile code.

The applications of the Wandering Network model for multimedia communications are numerous. For instance, adaptive QoS management and routing in ad-hoc mobile networks are some of them. Figure 1 illustrates the realization of a multifunctional netbot, proposed in the "Odysseus-2001" project, [14], a re-configurable MPEG-4 bit rate trancoding network proxy for a real-time dynamic UMTS QoS management in videoconferencing upon user feedback, [15]. 
Adaptive Media Agency Transcoding - Dynamic UMTS QoS Management -
variable bandwidth \& on-line in-band cell load adaptation upon user feedback

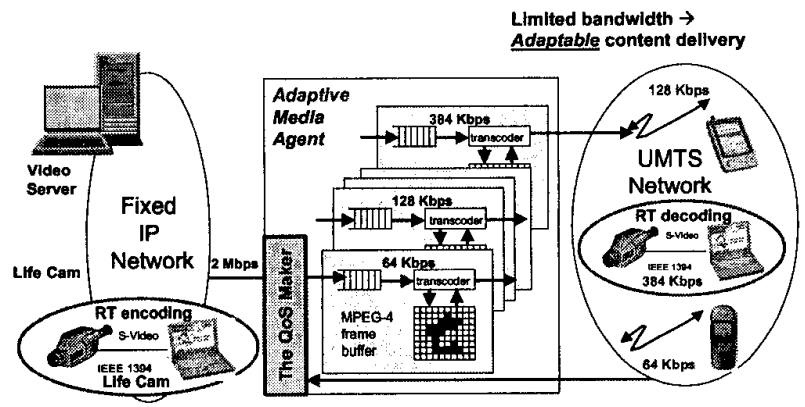

user feedback \& real time transcoding upon bandwidth adaptation

Figure 1: A WLI based adaptive media transcoder

\section{CONCLUSIONS}

The Wandering Network is a new type of an active ad-hoc mobile communications architecture for adaptive multimedia defined by the following characteristics, cf. [16]:

1. flexible, multi-modal specialization of network nodes as virtual subnetworks;

2. mobility and virtualization of the net functions as hardware und software;

3. self-organization as multi-feedback-based topology-on-demand and a tool of the user-oriented network evolution.

Network elemens in such an autonomous network contain different functional modules, and can therefore perform diverse network functions, e.g. depending on the actual user location and/or environment characteristics.

The essential contributions of the WLI approach and the resulting Wandering Network are summarized as follows:

1. Role Change: The role of the network node within a particular virtual architecture can change during its operation. The new functionality is either resident on the node and waiting to be activated, i.e. it is not yet involved in the next step virtual scheme, or transferred via Active Networking to the destination node.

2. Parallel Roles: The execution of the parts of a distributed algorithm can be performed within the different roles of an active node's, a netbot's, configuration.

3. Node Genesis ("N"-geneering): encoding and embedding the structural information about a mobile node, the netbot, and its environment into the executable part of the active packets, the shuttles. 


\section{BIBLIOGRAPHY}

1. P. L. Simeonov, "The Wandering Network, a Glance at an Evolving Reality" (in German: "Das Wandernde Netzwerk"), Proc. of Netobjectdays'2001, 2. Joint GI conference "Object Orieneted Programming for a Networked World", 11-13 Sept., 2001, Erfurt, Germany, http://www.prakinf.tu-ilmenau.de/IPI/FGT/.

2. D. Tennenhouse et al., "A Survey of Active Network Research", IEEE Comm. Mag., Vol. 35, No. 1, Jan. 1997, pp. 80-86.

3. K. L. Calvert, S. Bhattacharjee, E. Zegura, J. Sterbenz, "Directions in Active Networks," IEEE Communications Mag., Oct. 1998, pp. 72-78.

4. A. Campbell, H. De Meer, M. Kounavis, K. Miki, J. Vicente, D. Villela, "A Survey of Programmable Networks", http://www.columbia.comet.edu.

5. Humberto R. Maturana, Francisco J. Varela, "Autopoiesis and Cognition", D. Reidel Publishing Co., 1980, ISBN 9027710163.

6. K. Compton, S. Hauck, "Reconfigurable Computing: A Survey of Systems and Software", Northwestern University, Dept of ECE, Tech. Report, 1999.

7. DARPA Information Technology Office, "Adaptive Computing Systems Program ", http://www.darpa.mil/ito/acs.

8. O. Spaniol, J. Meggers, "Active Network Nodes for Adaptive Multimedia Communication", Proc. of SMARTNET'99, Nov. 1999.

$9 . \quad$ D. J. Wetherall, J. Guttag, D. L. Tennenhouse, "ANTS: A Toolkit for Building and Dynamically Deploying Network Protocols", Proc. of IEEE OPENARCH'98, San Francisco, CA, April 1998.

10. Simeonov, P. L., Hofmann, P., “A Distributed Intelligent Computer/Telephony Network Integration Architecture for Unified Media Communication", Proc. of $2 I N^{\prime} 97$, Paris, Sept. 2-5, 1997, pp. 3-8, Chapman \& Hall, ISBN 0-412-82950-9.

11. Simeonov, P. L., "The SmartNet Architecture or Pushing Networks beyond Intelligence", Proc. of ICIN'98, 12-15 May, Bordeaux, France, pp. 182-185.

12. Simeonov, P. L., "The Wandering Logic of Intelligence: Or Yet Another View on Nomadic Communications", Proc. of SMARTNET'99, 22-26 Nov. 1999, Asian Institute of Technology, Pathumthani, Thailand, pp. 293-306, Kluver Academic Publishers, ISBN: 0-7923-8691-1, http://www.cs.ait.ac.th/ ca/smartnet99/.

13. Jungmann, M., Paradies, Th., Reschke, D., "Component-oriented approach for hypermedia-based knowledge management applications", Proc.of IFMIP'98, Anchorage, Alaska, Mai 1998.

14. Simeonov, P. L. (Ed.), "MediaPEP, A Scaleable and Dependable ActiveUMTS QoS Management Server, An Internet Protocol Booster and An Adaptive Media Transcoder Switch Architecture for E2E-IP Integration of Mobile Wireless Interactive Telepresence Applications", White Paper, Siemens AG, Heinrich Hertz Institute, TU Berlin, TU Ilmenau, Northeastern University (Boston), Nov., 2000, http://www.prakinf.tu-ilmenau.de/IPI/FGT.

15. Hoang, D.-H., D. Reschke, W. Horn, "Adaptive Quality of Service Management Using QoS Proxy and User Feedback for Wireless Links", Workshop Innovative Internet Computing Systems, Juni 2001, LNCS 2060 (2001), Springer-Verlag, ISSN 0302-9743, pp. 31-40.

16. Simeonov, P. L., "The Viator Approach: About Four Principles of Autopoietic Growth On the Way to Hyperactive Network Architectures", 2002 IEEE International Symposium on Parallel \& Distributed Processing (IPDPS'02), April 15-19, 2002, Ft. Lauderdale, FL. 